\title{
A critical analysis on intrusion detection techniques
}

\author{
Sonal Wange ${ }^{1^{*}}$, Shiv K. Sahu ${ }^{2}$ and Amit Mishra ${ }^{3}$ \\ M.Tech Research Scholar, Department of Information Technology, TIT, Bhopal ${ }^{1}$ \\ Associate Professor and Head, Department of Information Technology, TIT, Bhopal ${ }^{2}$ \\ Assistant Professor, Department of Information Technology, TIT, Bhopal ${ }^{3}$
}

(C2016 ACCENTS

\begin{abstract}
An intrusion detection system (IDS) is an application platform that records system fuss for malicious activities and management of these activities. It is a malicious attack identification technique. It allows identifying Denial of service $(\mathrm{DoS})$, User to root $(\mathrm{U} 2 \mathrm{R})$, Remote to login (R2L) and Probe attack. There are several communication mechanism are rely on the networking today so the need of data security is important concern. The main aim of this paper is to focusing on improving attack detection rates. For this we have analyzed the KDD intrusion detection dataset. The main method for approaching this detection and analysis are data mining and optimization techniques for this study.
\end{abstract}

\section{Keywords}

Intrusion detection, DoS, U2R, R2L and Probe.

\section{Introduction}

Now a days there are special focus is on the detection of malicious behaviour in the network as all the business processing are relies on any type of network. The Association for Computing Machinery (ACM) has been gathered different network malicious and non-malicious behaviour data in a Knowledge Discovery and Data mining (KDD) platform [1] for the data mining understudies and experts. They have provided set KDD Cup99 data sets for network intrusion detection [2]. This gathering is use for intrusion recognition and a few specialists had considered this as the benchmark dataset for result examination.

As of late, various specialists are focusing to use data burrowing thoughts for Intrusion Detection [3]. This is a strategy to think the undeniable information and learning. Interruption disclosure is the philosophy of malignant ambush in the structure and framework when we are in no time correspondence or isolating data in the steady environment [4][5]. Since its development, interference area has been one of the key segments in fulfilling information security. It goes about as the second-line obstruction which supplements the passage controls.

*Author for correspondence
Exactly when the controls failed, the intrusion ID systems should have the ability to remember it consistent and alert the security officers to take incite and suitable exercises [5] [6].

Interference acknowledgment structure oversee managing the scenes happening in PC structure or framework circumstances and taking a gander at them for signs of possible events, which are infringement or certain perils to PC security, or standard security sharpens Intrusion recognizable proof systems (IDS) have ascended to recognize exercises which imperil the uprightness, protection or availability of are source as a push to give a response for existing security issues [7-10]. So in the above course we ponder a couple of points of view in the subsequent sections. We similarly discuss data mining and headway procedures, in light of the fact that it can be used as a piece of forming the structure which conveys better distinguishing proof system. As we are inspected this study toward a prevalent framework with the blend of data mining and streamlining. These systems are useful and has been used as a piece of differing procedures like [11-14]. So the use of these estimations can enhance an impact.

\section{Literature survey}

In 2012, P. Prasenna et al. [15] suggested that in standard framework security just relies on upon numerical computations and low counter measures to 
taken to turn away intrusion recognizable proof system, but the lion's share of this approachs to the extent theoretically tried to execute. Makers recommend that instead of delivering generous number of standards the progression change methods like Genetic Network Programming (GNP) can be used .The GNP is in perspective of composed chart. They focus on the security issues related to send a data mining-based IDS in a consistent circumstance. They total up the issue of GNP with connection standard mining and propose a feathery weighted association rule mining with GNP framework suitable for both consistent and discrete qualities.

In 2011, LI Han [16] focuses on interference disclosure in light of collection examination. The fact of the matter is to improve the acknowledgment rate and decrease the false alert rate. A balanced component K-suggests count called MDKM to recognize irregularity activities is proposed and relating reenactment investigations are presented. Firstly, the MDKM figuring channels the upheaval and isolated spotlights on the data set. Additionally by finding out the divisions between all illustration data centers, they secure the high-thickness parameters and gathering part parameters, using component iterative strategy we get the k clustering concentrate exactly, then a peculiarity revelation model is shown. They used KDD CUP 1999 data set to test the execution of the model. Their results exhibit the structure has a higher acknowledgment rate and a lower false alert rate, it accomplishes confident point.

In 2011, Z. Muda et al. [17] discuss the issue of current anomaly recognizable proof that it not ready to recognize an extensive variety of ambushes viably. To beat this issue, they propose a crossbreed learning approach through mix of K-Means bundling and Naïve Bayes portrayal. The proposed philosophy will be gathering all data into the looking at assembling before applying a classifier for request reason. An examination is done to evaluate the execution of the proposed technique using KDD Cup '99 dataset. Come about exhibit that the proposed philosophy performed better in term of precision, area rate with sensible false alert rate.

In 2014, Deshmukh et al. [18] introduces a Data Mining framework in which distinctive preprocessing procedures will be incorporated, for example, Normalization, Discretization and Feature decision. With the assistance of these strategies the data will be preprocessed and obliged highlights are picked. They used NaIve Bayes framework in coordinated learning methodology which assembles distinctive framework events for the KDD cup'99 Dataset.

In 2014, Benaicha et al. [19] show a Genetic Algorithm (GA) approach with an upgraded beginning masses and decision executive, to capably distinguish diverse sorts of framework intrusions. They used GA to improve the look of ambush circumstances in audit reports, on account of its awesome balance examination/abuse; according to the makers it gives the subset of potential attacks which are show in the survey archive in a sensible get ready time. The testing time of the Network Security Laboratory Knowledge Discovery and Data Mining (NSL-KDD99) benchmark dataset has been used to recognize the mishandle works out. Their system of IDS with Genetic estimation increases the execution of the recognizable proof rate of the Network Intrusion Detection Model and declines the false positive rate.

In 2014 Kiss et al. [20] suggest that Modern Networked Critical Infrastructures (NCI), including advanced and physical systems, are introduced to sharp computerized attacks concentrating on the unfaltering operation of these structures. To ensure variation from the norm care, their watched data can be used as a piece of concurrence with data mining methods to make Intrusion Detection Systems (IDS) or Anomaly Detection Systems (ADS). They proposed a gathering based philosophy for recognizing advanced strikes that cause idiosyncrasies in NCI. Distinctive clustering methodologies are examined to pick the most suitable for gathering the time-course of action data highlights, hence portraying the states and potential computerized attacks to the physical structure. The Hadoop execution of MapReduce standard is used to give a suitable planning environment to broad datasets.

In 2014, Thaseen et al. [21] proposed a novel procedure for organizing crucial fragment examination (PCA) and support vector machine (SVM) by overhauling the piece parameters using customized parameter determination framework. Their procedure diminishes the arrangement and testing time to recognize interferences therefore upgrading the precision. Their proposed methodology was attempted on KDD data set. The datasets were carefully parceled into planning and testing considering the minority ambushes, for instance, $\mathrm{U} 2 \mathrm{R}$ and R2L to be display in the testing set to 
recognize the occasion of cloud strike. Their results demonstrate that the proposed procedure is viable in perceiving interferences. Their exploratory results exhibit that the request precision of the proposed framework defeats other course of action methodologies using SVM as the classifier and other dimensionality lessening or highlight decision frameworks.

In 2014, Wagh et al. [22] proposed Network security is a key a portion of web enabled systems in the present world circumstance. According to the makers as a result of confusing chain of PCs the open entryways for intrusions and strikes have extended. Along these lines it is need of extraordinary significance to find the best courses possible to secure our structures. So the makers propose intrusion distinguishing proof structure is expecting essential part for PC security. The best methodology used to handle issue of IDS is machine learning. Thy watched that the rising field of semi managed learning offers an ensured course to correspond investigation. So they proposed a semi-oversaw framework to reduce false ready rate and to improve revelation rate for IDS.

In 2014, Masarat et al. [23] displayed a novel multistep structure considering machine learning methodology to make a capable classifier. In initial step, the highlight decision strategy will execute considering get extent of highlights by the makers. Their system can upgrade the execution of classifiers which are made considering these highlights. In classifiers blend step, we will show a novel soft assembling method. Along these lines, classifiers with more execution and lower cost have more effect to make the last classifier.

In 2015, Yan et al. [24] focusing at false negative rate and false alarm rate which exist generally in the intrusion detection system. They have proposed an intelligent intrusion detection model. Based on the characteristics of global superiority of genetic algorithm and locality of nerve, the model optimizes the weights of the neural network using genetic algorithm. Their experiment results show that the intelligent way can improve the efficiency of the intrusion detection.

\section{Problem identification}

Based on the study and review work the following observations have been made:

1) Results can be checked with different variations of data.

2) Optimization based classification is missing.

3) Training based data classification can be used as the distributed classifier.

4) All type of attacks is not well detected.

5) Maintain long $\log$ file for detection.

6) K-Means with K-Nearest Neighbor (KMKNN) approach can be applied for better intrusion detection.

7) The data mining combination with evolutionary approach can be a better combination as it is efficient in finding high and low thresholds.

8) Probability of less detection in U2R and R2L detection technique so there is the need of a detection technique which improves in the hybridization of above two.

\section{Analysis}

After studying and observing several research works we compare the result discussions by their techniques, so that we identify the weak attack detection area. The results comparison of different methods is shown in Table 1.

Table 1 Analysis

\begin{tabular}{|c|c|c|c|c|}
\hline S.No & Approach & Accuracy $(\%)$ & Advantage & Disadvantage \\
\hline 1 & $\begin{array}{l}\text { Intrusion } \\
\text { detection system } \\
\text { using genetic } \\
\text { algorithm and } \\
\text { fuzzy logic [25] }\end{array}$ & NA & $\begin{array}{l}\text { Suggest that the combination is better in } \\
\text { classifying the results. }\end{array}$ & $\begin{array}{l}\text { A practical implication of this } \\
\text { approach is missing. }\end{array}$ \\
\hline 2 & $\begin{array}{l}\text { Layer wise } \\
\text { Individual Feature } \\
\text { Set [26] }\end{array}$ & $\begin{array}{l}\text { DOS-97.8 U2R- } \\
88.2 \quad \text { R2L-30.2 } \\
\text { Probe-98.6 }\end{array}$ & $\begin{array}{l}\text { They have used Individual Feature Set and } \\
\text { Layer wise approach to achieve the } \\
\text { efficiency and accuracy in detecting the } \\
\text { network attack group. }\end{array}$ & $\begin{array}{l}\text { There is the need of } \\
\text { improvement in the results. }\end{array}$ \\
\hline 3 & $\begin{array}{l}\text { Neural Network } \\
\text { Classifier [27] }\end{array}$ & $\begin{array}{l}\text { Overall accuracy } \\
85.56 .\end{array}$ & $\begin{array}{l}\text { The study suggests probabilistic neural } \\
\text { networks provide better accuracy over Feed } \\
\text { Forward Neural Network. }\end{array}$ & $\begin{array}{l}\text { Need to include the feature } \\
\text { reduction techniques to } \\
\text { improve the efficiency. }\end{array}$ \\
\hline 4 & $\mathrm{GA}+\mathrm{SVM}[28]$ & $\begin{array}{l}\text { Overall accuracy } \\
98.33 \text {. }\end{array}$ & They have used hybrid SVM using GA. & $\begin{array}{l}\text { Need to classify each } \\
\text { separately so that the }\end{array}$ \\
\hline
\end{tabular}




\begin{tabular}{|c|c|c|c|c|}
\hline S.No & Approach & Accuracy (\%) & Advantage & Disadvantage \\
\hline 5 & $\begin{array}{l}\text { Clustering and } \\
\text { Outlier } \\
\text { Detection[29] }\end{array}$ & $\begin{array}{l}\text { Overall accuracy } \\
97.77 .\end{array}$ & $\begin{array}{l}\text { They have used supervised and outlier } \\
\text { methods }\end{array}$ & $\begin{array}{l}\text { classification can be } \\
\text { understood easily. } \\
\text { More ensemble approach is } \\
\text { needed for the classification. }\end{array}$ \\
\hline 6 & Decision Tree [30] & $\begin{array}{l}\text { Overall accuracy } \\
94.74 .\end{array}$ & $\begin{array}{l}\text { They have applied decision tree with } \\
\text { sampling. }\end{array}$ & $\begin{array}{l}\text { There is the need of } \\
\text { improvement in the results. }\end{array}$ \\
\hline
\end{tabular}

\section{Conclusion and future work}

This paper provides a direction in the face of Intrusion detection improvement. Our study suggests the following gaps which can be considered as the future directions:

1) A data mining technique like the combination of association rule mining and evolutionary algorithm can increase the pattern recognition.

2) Particle swarm optimization or Ant Colony optimization is also helpful as it is better in threshold determination.

3) The combination of positive and negative association rule can be better as they are efficient in lower and higher value support determination.

\section{Acknowledgment}

None.

\section{Conflicts of interest}

The authors have no conflicts of interest to declare.

\section{References}

[1] Tarakanov AO, Kvachev SV, Sukhorukov AV. A formal immune network and its implementation for on-line intrusion detection. In MMM-ACNS 2005 (pp. 394-405).

[2] Farhaoui Y. How to secure web servers by the intrusion prevention system (IPS)? International Journal of Advanced Computer Research. 2016; 6(23); 65-71.

[3] Jianliang M, Haikun S, Ling B. The application on intrusion detection based on k-means cluster algorithm. In international forum on information technology and applications 2009 (pp. 150-2). IEEE.

[4] Lundin E, Jonsson E. Survey of Intrusion Detection Research. 2002.

[5] Venkatesan R, Ganesan R, Selvakumar AA. A comprehensive study in data mining frameworks for intrusion detection. International Journal of Advanced Computer Research. 2012; 2(7):29-34.

[6] Devaraju S, Ramakrishnan S. Performance analysis of intrusion detection system using various neural network classifiers. In international conference on recent trends in information technology 2011 (pp. 1033-8). IEEE.

[7] Ishida M, Takakura H, Okabe Y. High-performance intrusion detection using optigrid clustering and gridbased labelling. In international symposium on applications and the internet (SAINT), IEEE/IPSJ
2011 (pp. 11-9). IEEE.

[8] Brugger ST. Data mining methods for network intrusion detection. University of California at Davis. 2004.

[9] Conteh NY, Schmick PJ. Cybersecurity: risks, vulnerabilities and countermeasures to prevent social engineering attacks. 2016; 6(23):31-8.

[10] Nalavade K, Meshram BB. Mining association rules to evade network intrusion in network audit data. International Journal of Advanced Computer Research. 2014; 4(15):560-7.

[11] Lee W, Stolfo SJ. Data mining approaches for intrusion detection. In Usenix security 1998.

[12] Naoum R, Aziz S, Alabsi F. An enhancement of the replacement steady state genetic algorithm for intrusion detection. International Journal of Advanced Computer Research. 2014; 4(15):487-93.

[13] Shrivastava A, Baghel M, Gupta H. A review of intrusion detection technique by soft computing and data mining approach. International Journal of Advanced Computer Research. 2013; 3(12):224-8.

[14] Yin-huan LI. Design of intrusion detection model based on data mining technology. In 2012 international conference on industrial control and electronics engineering 2012.

[15] Prasenna P, RaghavRamana AV, Krishnakumar R, Devanbu A. Network programming and mining classifier for intrusion detection using probability classification. In international conference on pattern recognition, informatics and medical engineering (PRIME) 2012 (pp. 204-9). IEEE.

[16] Han LI. Using a dynamic K-means algorithm to detect anomaly activities. In seventh international conference computational intelligence and security (CIS) 2011(pp. 1049-52). IEEE.

[17] Muda Z, Yassin W, Sulaiman MN, Udzir NI. Intrusion detection based on k-means clustering and naïve bayes classification. In international conference on information technology in Asia (CITA 11) 2011(pp. 16). IEEE.

[18] Deshmukh DH, Ghorpade T, Padiya P. Intrusion detection system by improved preprocessing methods and naïve bayes classifier using NSL-KDD 99 Dataset. In international conference on electronics and communication systems (ICECS) 2014 (pp. 1-7). IEEE.

[19] Benaicha SE, Saoudi L, Guermeche B, Eddine S, Lounis O. Intrusion detection system using genetic algorithm. In IEEE science and information conference (SAI) 2014 (pp. 564-68). 
[20] Kiss I, Genge B, Haller P, Sebestyen G. Data clustering-based anomaly detection in industrial control systems. In IEEE international conference on intelligent computer communication and processing (ICCP) 2014 (pp. 275-81). IEEE.

[21] Thaseen IS, Kumar CA. Intrusion detection model using fusion of PCA and optimized SVM. In international conference on contemporary computing and informatics (IC3I) 2014 (pp. 879-84). IEEE.

[22] Wagh SK, Kolhe SR. Effective intrusion detection system using semi-supervised learning. In international conference on data mining and intelligent computing (ICDMIC) 2014 (pp. 1-5). IEEE.

[23] Masarat S, Taheri H, Sharifian S. A novel framework based on fuzzy ensemble of classifiers for intrusion detection systems. In international conference on computer and knowledge engineering (ICCKE) 2014 (pp. 165-70). IEEE.

[24] Yan C. Intelligent Intrusion Detection Based on Soft Computing. In seventh international conference on measuring technology and mechatronics automation (ICMTMA) 2015 (pp. 577-80). IEEE.

[25] Hassan MM. Current studies on intrusion detection system, genetic algorithm and fuzzy logic. arXiv preprint arXiv:1304.3535. 2013.
[26] Manivannan SS, Sathiyamoorthy E. An efficient and accurate intrusion detection system to detect the network attack groups using the layer wise individual feature set. International Journal of Engineering and Technology. 2013; 5(4):3243-50.

[27] Devaraju S, Ramakrishnan S. Detection of accuracy for intrusion detection system using neural network classifier. International Journal of Emerging Technology and Advanced Engineering. 2013; 3(1): 338-45.

[28] Atefi K, Yahya S, Dak AY, Atefi A. A hybrid intrusion detection system based on different machine learning algorithms. In international conference on computing and informatics 2013 (pp. 312-320).

[29] Jeyanna JA, Indumathi E, Punithavathani DS. A network intrusion detection system using clustering and outlier detection. International Journal of Innovative Research in Computer and Communication Engineering. 2015; 3(2):975-82.

[30] Kailashiya D, Jain RC. Improve intrusion detection using decision tree with sampling. International Journal of Computer Technology and Applications. 2012;3(3): 1209-16. 